\title{
Calculating Quark Number Susceptibilities with Domain-Wall Fermions
}

\section{Prasad Hegde $^{*, a}$, Frithjof Karsch ${ }^{b, c}$, Christian Schmidt $^{c}$ (RBC Collaboration)}

${ }^{a}$ Dept. of Physics and Astronomy, SUNY Stony Brook, Stony Brook, NY 11790, USA.

E-mail: phegde@quark •phy . bn l.gov

${ }^{b}$ Department of Physics, Brookhaven National Laboratories, Upton, NY 11733, USA.

E-mail: karsch@quark.phy.bnl.gov

${ }^{c}$ Fakultät für Physik, Universität Bielefeld, D-33615 Bielefeld, Germany.

E-mail: schmidtephysik.uni-bielefeld.de

We present results from calculations of different quark number and hadronic susceptibilities on $2+1$-flavor dynamical domain wall ensembles. We find that the iso-spin and electric charge susceptibilities are especially well suited to determine the transition temperature, as these quantities show only small statistical errors. Moreover, the transition values of the coupling obtained from iso-spin and electrical charge susceptibilities are in good agreement with the one obtained from the chiral condensate.

The XXVI International Symposium on Lattice Field Theory

July 14-19 2008

Williamsburg, Virginia, USA

${ }^{*}$ Speaker. 


\section{Introduction}

Sufficiently hot and dense hadronic matter undergoes a transition from a confined hadronic phase to a deconfined, chirally symmetric medium, the quark gluon plasma (QGP). At zero chemical potential, the transition has been established by lattice QCD simulations, which have proven to be a powerful method to analyze the non-perturbative features of the QGP close to the transition region. At non-zero chemical potential, however, lattice simulations are limited by the sign problem. In fact, direct simulations by standard Monte Carlo methods are not possible. In order to evaluate the response of the medium to a small non-zero chemical potential, we perform a Taylor expansion of the pressure (grand canonical potential) [1]. For the dimensionless combination $p / T^{4}$ we define the expansion coefficients $c_{i j k}$ as

$$
\frac{p}{T^{4}}-\left.\frac{p}{T^{4}}\right|_{\mu=0}=\sum_{i, j, k} c_{i j k}\left(\frac{\mu_{u}}{T}\right)^{i}\left(\frac{\mu_{d}}{T}\right)^{j}\left(\frac{\mu_{s}}{T}\right)^{k} .
$$

Here $\mu_{u, d, s}$ are the chemical potentials of up-, down- and strange-quarks, respectively. The coefficients $c_{i, j, k}$ have been calculated previously using staggered [2,3,4,5] and Wilson [6] quarks. As they are evaluated at vanishing chemical potential they also provide important information about thermodynamic properties of QCD at vanishing baryon number density.

We present here preliminary results on the smallest non-vanishing coefficients ${ }^{1}$ which are $c_{200} \equiv c_{2}^{u}, c_{002} \equiv c_{2}^{s}, c_{110} \equiv c_{11}^{u d}$ and $c_{101} \equiv c_{11}^{u s}{ }^{2}$ Apart from these basic coefficients characterizing fluctuations and correlations for different quark flavors, we consider combinations of them that yield iso-spin (I) and electric charge (Q) fluctuations,

$$
\begin{aligned}
& c_{2}^{I}=\frac{1}{4}\left(2 c_{2}^{u}-c_{11}^{u d}\right), \\
& c_{2}^{Q}=\frac{1}{9}\left(5 c_{2}^{u}+c_{2}^{s}-2 c_{11}^{u d}-c_{11}^{u s}\right) .
\end{aligned}
$$

In general, the second order coefficients are related to the quadratic fluctuations, also known as susceptibilities $\chi_{2}^{X}$, of the corresponding charge densities $\left(n_{X}\right)$. Fourth order coefficients are related to the quartic fluctuations $\chi_{4}$. In terms of the expansion coefficients we have

$$
\begin{aligned}
& \frac{\chi_{2}^{X}}{T^{2}} \equiv\left\langle n_{X}^{2}\right\rangle=2 c_{2}^{X}, \\
& \frac{\chi_{4}^{X}}{T^{2}} \equiv\left\langle n_{X}^{4}\right\rangle-3\left\langle n_{X}^{2}\right\rangle^{2}=24 c_{4}^{X} \quad \text { with } \quad X \in\{u, d, s, I, Q, \ldots\} .
\end{aligned}
$$

In Fig. 1, we show results for $c_{2}^{X}$ and $c_{4}^{X}$ for $X=u, s$ calculated with staggered fermions [3]. It is apparent that $c_{2}^{X}$ varies rapidly and $c_{4}^{X}$ peaks within a small temperature range. This behavior may be interpreted as signaling a transition from a confined phase consisting of heavy hadrons to a deconfined phase consisting of partons. In fact, the ratio $\chi_{4}^{(X)} / \chi_{2}^{(X)}$ is directly related to the squared unit charge of quantum number $X$, for the relevant degrees of freedom [7].

The phase diagram of the QGP at $\mu=0$ is by now believed to be understood quite well. E.g., we have plenty of evidence that the transition is a sharp crossover, rather than a true phase

\footnotetext{
${ }^{1}$ It can be shown that the odd-order coefficients vanish through $\mathrm{CP}$-symmetry.

${ }^{2}$ In the 2+1-flavor theory up and down quark masses are degenerate. The coefficients $c_{i j k}$ and $c_{j i k}$ are thus equal.
} 

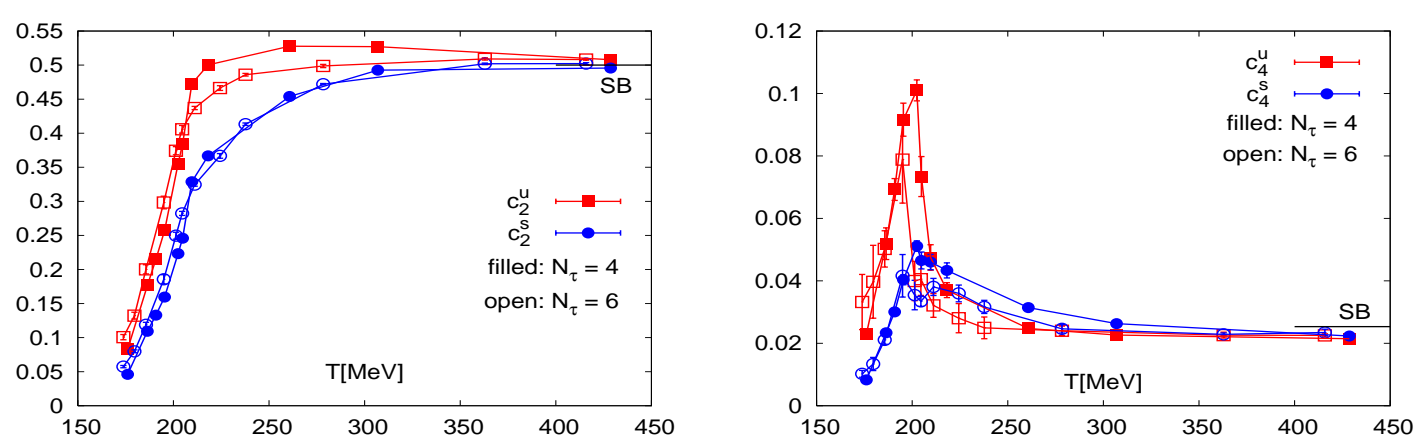

Figure 1: Staggered quark results for the Taylor coefficients $c_{2}^{u, s}$ and $c_{4}^{u, s}$, obtained with (2+1)-flavor of p4fat3 fermions on $N_{\tau}=4$ and 6 lattices [3].

transition. Hence, the general behavior of $c_{2}$ and $c_{4}$ as shown in Fig. 1 will not change, even in the thermodynamic limit. They may, however be sensitive to the values of the light quark masses. At vanishing light quark mass, the transition at $\mu=0$ is expected to be second order, in the same universality class as the three-dimensional $O(4)$-models. In this case the appropriate scaling field $t$ (reduced temperature) in the vicinity of the phase transition [8] depends on a combination of $T$ and $\mu$

$$
t=\left|\frac{T-T_{c}}{T_{c}}\right|+A\left(\frac{\mu}{T_{c}}\right)^{2}
$$

This explains the general structure of the temperature dependence of $c_{2 n}$. We thus may expect that the behavior of the $(2 n)$-th $\mu$-derivative of the partition function is similar to that of the $n$ th temperature derivative; e.g. $\varepsilon \sim \partial \ln Z / \partial T \Leftrightarrow c_{2} \sim \partial^{2} \ln Z / \partial \mu^{2}$. A plot of $c_{2}^{X}$ versus the temperature thus shall resemble the corresponding plot for the energy density, while that of $c_{4}^{X}$ shall resemble the specific heat $C_{v}$.

We expect this general pattern of the expansion coefficients also to be reproduced in calculations with domain wall fermions. Details of the temperature dependence, in particular at low temperature, may, however, be sensitive to chiral properties of the lattice discretization scheme used. Our main interest in performing these calculations also with domain wall fermions thus is to understand to what extent the better chiral symmetry of this fermion action shows up in thermodynamic observables that are directly sensitive to the hadron spectrum.

\subsection{Beyond the Staggered Formulation}

Chiral symmetry plays an important role in determining the nature of the QGP transition. In the staggered formulation, the full $S U(3)_{L} \times S U(3)_{R}$ chiral symmetry is explicitly broken; at nonzero values of the lattice spacing only two $U(1)$ subgroups remain; one in the light and strange quark sector, respectively. As a consequence universal properties at the chiral phase transition (in the chiral limit) may be recovered only in the continuum limit. Moreover, the loss of full chiral symmetry leads to too few light degrees of freedom at low temperatures $e$.g. one light pion instead of three, which may influence bulk thermodynamic properties in this regime. Furthermore, questions have been raised about the validity of "the rooting trick" used for staggered fermions [9], which at present are not fully settled [10, 11]. 
It is clearly desirable to go beyond the staggered formulation and work with a fully chiral formulation. Domain Wall Fermions (DWF) meet these criteria [12]. In this formulation, one introduces a fifth dimension $(x, y, z, t, s)$, so that the lattice dimensions are $N_{\sigma}^{3} \times N_{\tau} \times L_{s}$. Only fermions can propagate along the fifth direction; the gauge fields exist only on the 4-dimensional slices $s=0,1, \ldots L_{s}-1$. For this reason, the Domain Wall formulation can also be thought of as a theory of $L_{s}$ fermion flavors coupled in a nontrivial way. When one simulates the theory, one finds, among other massive modes, two solutions that satisfy [13]

$$
\left[M_{d w f}(p)\right]_{s, s^{\prime}} \Psi_{s^{\prime}}^{( \pm)}(p)=\left(-i \sum_{\mu=1}^{4} \sin ^{2} p_{\mu}\right) \Psi_{s}^{( \pm)}(p),
$$

where $M_{d w f}$ is the Domain-Wall matrix and a sum over $s^{\prime}$ is implied. Furthermore, these modes are eigenstates of $\gamma_{5}$ and their wavefunctions are found to be localized on the two hyper-planes $s=0$ and $s=L_{s}-1$ respectively. These are exactly the chiral modes we want. Using a Pauli-Villars subtraction allows to remove contributions of the heavy modes [14] which leaves us with a theory that is, for $L_{s} \rightarrow \infty$, chiral-invariant under the full symmetry group. At finite values of $L_{s}$ a socalled residual mass leads to violations of chiral symmetry. In practice one is forced to perform calculations at small lattice spacings to suppress these residual mass effects. For thermodynamics calculations this means that calculations should be performed for $N_{\tau} \geq 8$ and/or sufficiently large $L_{s}$ to suppress the residual mass effects. In fact, the calculations we present here only marginally satisfy these constraints [15] and thus should be considered as a first feasibility study.

\section{Details of the Simulation}

We use here finite temperature 2+1-flavor dynamical domain-wall fermion ensembles generated by the RBC-Collaboration [15]. The lattice dimensions are $16^{3} \times 8 \times 32$. The light and strange quark masses used in these calculations are $a m_{q}=0.003$ and $a m_{s}=0.037$, and the domain-wall height and anisotropy factor were $a M_{5}=1.8$ and $a / a_{5}=1.00$ respectively. The gauge configurations have been generated at several different values of the coupling, ranging from $\beta=1.95$ to $\beta=2.14$. This covers the transition region from low to high temperatures. On these data sets we started to calculate the lowest order expansion coefficients.

The expansion coefficients can entirely be expressed in terms of traces of the (5-dimensional) DWF fermion matrices over space-time, color and spin indices. For the diagonal and off-diagonal coefficients we obtain from derivatives of the fermion matrices for light $\left(M_{u}\right)$ and strange $\left(M_{s}\right)$ quarks,

$$
c_{2}^{X}=\frac{1}{2 V T}\left\{\left\langle\operatorname{tr}\left(M^{-1} \frac{\mathrm{d}^{2} M}{\mathrm{~d} \mu_{X}^{2}}\right)\right\rangle-\left\langle\operatorname{tr}\left(M^{-1} \frac{\mathrm{d} M}{\mathrm{~d} \mu_{X}} M^{-1} \frac{\mathrm{d} M}{\mathrm{~d} \mu_{X}}\right)\right\rangle+\left\langle\operatorname{tr}^{2}\left(M^{-1} \frac{\mathrm{d} M}{\mathrm{~d} \mu_{X}}\right)\right\rangle\right\}
$$

and

$$
c_{11}^{X Y}=\frac{1}{V T}\left\langle\operatorname{tr}\left(M^{-1} \frac{\mathrm{d} M}{\mathrm{~d} \mu_{X}}\right) \operatorname{tr}\left(M^{-1} \frac{\mathrm{d} M}{\mathrm{~d} \mu_{Y}}\right)\right\rangle,
$$

respectively. Here we used the shorthand notation $M \equiv M_{X}$ for $X=u$, s. We estimated the traces using the stochastic random noise method with around $100-150$ random vectors for each trace. 
Products of traces were evaluated in an unbiased manner. Some simulation details and our current statistics are summarized in Table 2.

\begin{tabular}{|l|c|c|c|}
\hline \multicolumn{1}{|c|}{$\beta$} & \# conf. & separation & \# r.v. \\
\hline 2.14 & 40 & 10 & 100 \\
2.11 & 35 & 10 & 100 \\
2.0625 & 111 & 10 & 150 \\
2.05 & 81 & 25 & 150 \\
2.0375 & 96 & 20 & 150 \\
2.025 & 71 & 20 & 150 \\
2.0125 & 125 & 10 & 150 \\
1.975 & 61 & 10 & 150 \\
1.95 & 73 & 10 & 150 \\
\hline
\end{tabular}

Table 1: Details of the calculation: The columns give from left to right the values of the lattice coupling $\beta$, the number of evaluated configurations, the number of trajectories by which these configurations are separated and the number of random vectors used for the evaluation of the traces.

\section{Results}

A word about the systematic errors: It can be shown that domain wall fermions satisfy the same dispersion relation as naive fermions [16]. This implies that thermodynamic quantities computed with the standard DWF action shall have $\mathscr{O}\left(a^{2}\right)$ cut-off errors that are of the same magnitude as those of a standard staggered or naive fermion discretization scheme. This translates to $\sim 10 \%$ cutoff errors at $N_{\tau}=8$ [17]. Remarkably, the above continues to be true even after the introduction of a chemical potential, with only the magnitude of the correction changing [17, 18]. We note in passing that these $\mu$-dependent cut-off corrections can be computed exactly in the ideal gas limit; they are given by Bernoulli polynomials.

The statistical error in the susceptibilities was determined by the jackknife method. There are two independent contributions to the error: The error due to the finite size of the ensemble and the error in the trace due to the finite number of random vectors. The latter was found to be especially severe for the "disconnected" contributions, which are the products of traces in Eqs. (2.1) and (2.2). This shows up in the large error bars for the coefficients $c_{2}^{u}, c_{2}^{s}$ and especially the offdiagonal coefficients $c_{11}^{u d}$ and $c_{11}^{u s}$, as can be seen from Fig. 2. Although $c_{2}^{u}$ and $c_{2}^{s}$ do transit from a low value to a high one, it is difficult to assign a corresponding transition temperature or equivalent a coupling $\beta=\beta_{d}$.

The disconnected contributions completely or partially cancel each other in the iso-spin and electric charge expansion coefficients $c_{2}^{I}$ and $c_{2}^{Q}$. This results in much smaller errors for these two quantities as can be seen in Fig. 3. The coefficients $c_{2}^{I}$ and $c_{2}^{Q}$ show a smooth transition from a low to a high value. There is some indication that they will overshoot the Stefan-Boltzmann (SB) ideal gas values at higher temperatures. This would be expected from the analysis of cut-off effects in thermodynamics within the DWF formulation [17]. This deserves further analysis. 

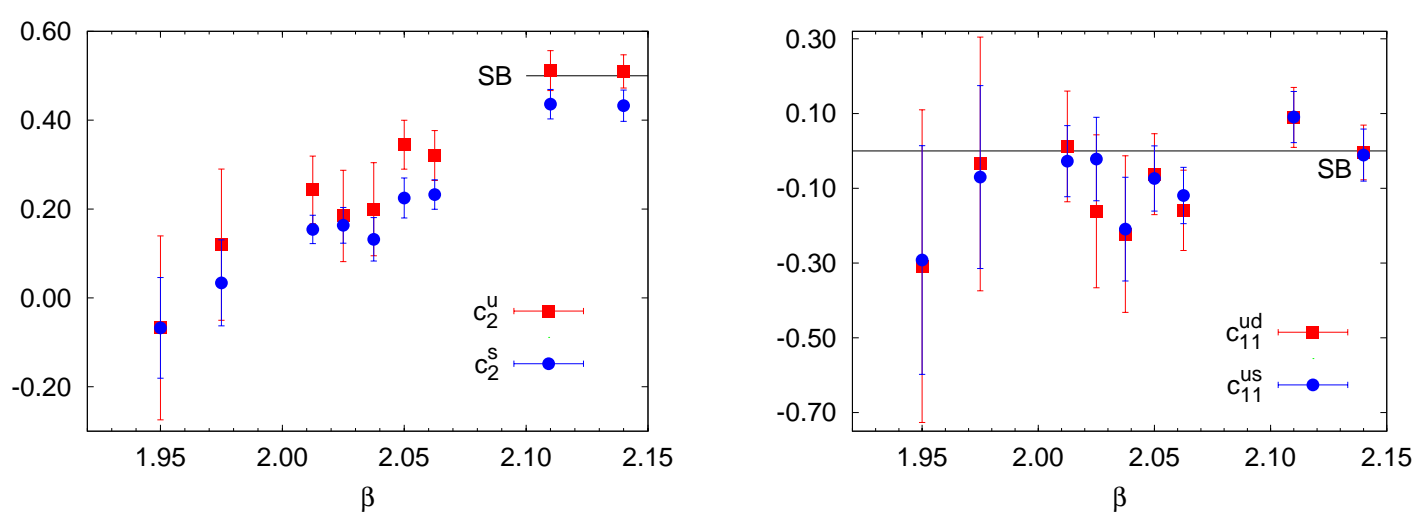

Figure 2: The expansion coefficients $c_{2}^{u}, c_{2}^{s}$ (left) and $c_{11}^{u d}, c_{11}^{u s}$ (right) as a function of the coupling $\beta$.

As we do, at present, have no results on higher order expansion coefficients, e.g. $c_{4}^{X}$, which could directly be used to identify a pseudo-critical coupling from the location of a peak in $c_{4}^{X}$, we estimate a pseudo-critical coupling, $\beta=\beta_{d}$, from fits of $c_{2}^{(Q, I)}$. We use the ansatz [13]

$$
c_{2}^{(Q, I)}=A \tanh \left\{B\left(\beta-\beta_{d}\right)\right\}+C .
$$

The resulting curves are also shown in Fig. 3, superimposed on the data points. Table 3 contains the best fit values of the free parameters $A, B, C$ and $\beta_{d}$.

For the transition value of the coupling we obtain $\beta_{d}=2.028(6)\left(\beta_{d}=2.030(7)\right)$ from the fit to $c_{2}^{I}\left(c_{2}^{Q}\right)$. This value is in agreement with the value $\beta_{c}=2.031(5)$ obtained by the RBCcollaboration [15] from an analysis of the chiral condensate. We thus find that the deconfinement transition and chiral symmetry restoring transition occur in the same narrow temperature interval.

From a determination of the Sommer parameter, $\left.r_{0} a^{-1}\right|_{\beta=\beta_{d}}=3.25(18)$, the group was also able to deduce $T_{c}=171(10)(17) \mathrm{MeV}$ [15]. While the latter error arose from the chiral and continuum extrapolations, the former was due to the fact that the residual mass varied across the temperature range and was significantly large for $\beta \lesssim \beta_{c}$.
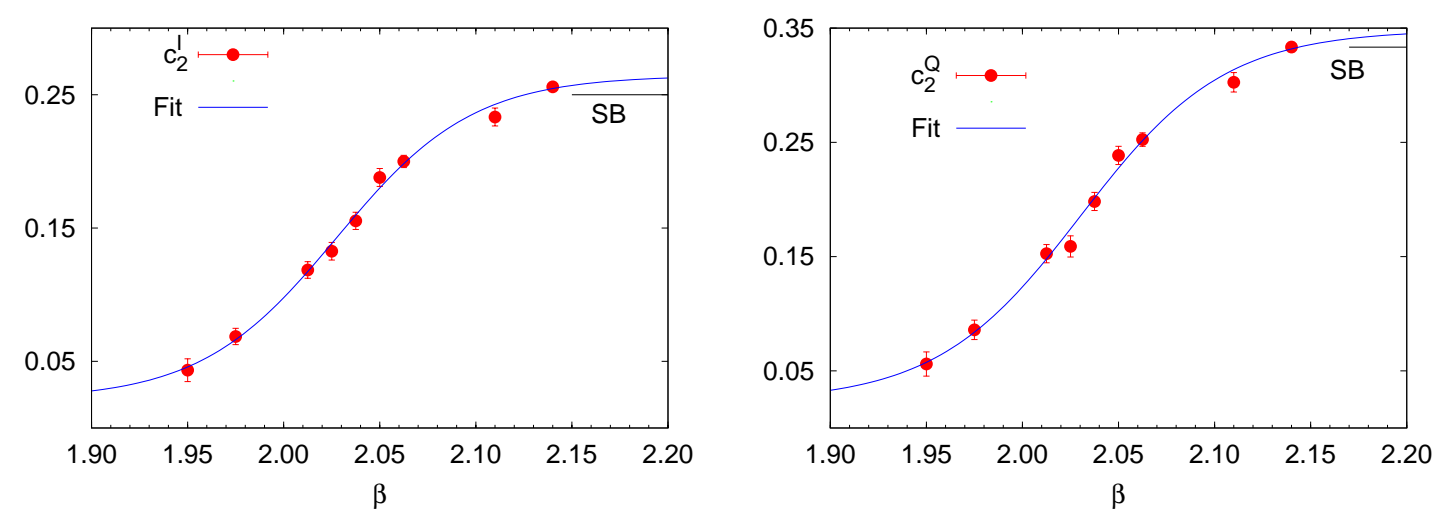

Figure 3: $c_{2}^{I}$ and $c_{2}^{Q}$ as function of the lattice coupling $\beta$. The smooth curve is a fit of the ansatz given in Eq. 3.1 to the data; the best fit values are given in Table 3 . 


\begin{tabular}{|c|c|c|c|c|c|}
\hline & $A$ & $B$ & $C$ & $\beta_{d}$ & $\chi^{2} / \mathrm{dof}$ \\
\hline$c_{2}^{I}$ & $0.49(5)$ & $14.1(2.3)$ & $0.57(3)$ & $\mathbf{2 . 0 2 8}(\mathbf{6})$ & 1.028 \\
\hline$c_{2}^{Q}$ & $0.16(2)$ & $13.3(2.7)$ & $0.19(1)$ & $\mathbf{2 . 0 3 0}(7)$ & 1.418 \\
\hline
\end{tabular}

Table 2: The best fit values and their errors for the fit of $c_{2}^{(I, Q)}$ to the ansatz given in Eq. 3.1.

We have stressed earlier that the current analysis is exploratory and that, in particular, the residual mass effects inherent in the DWF ansatz need to be reduced through calculations performed for large values of $L_{s}$ and/or $N_{\tau}$. Clearly, this requires a more thorough analysis in the future.

\section{Acknowledgments}

We thank Chulwoo Jung and Michael Cheng for helpful discussions. The numerical simulations were performed on the BlueGene/L computer at the New York Center for Computational Science (NYCCS). The work of FK and PH has been supported under Contract No. DE-AC0298CH10886 of the U.S. Department of Energy.

\section{References}

[1] C. R. Allton et al., Phys. Rev. D 66, 074507 (2002) [arXiv:hep-lat/0204010].

[2] C. R. Allton et al., Phys. Rev. D 71, 054508 (2005) [arXiv:hep-lat/0501030].

[3] C. Miao and C. Schmidt, Proceedings of this conference.

[4] R.V. Gavai and S. Gupta, [ArXiv:0806.2233 [hep-lat]]; S. Gupta, Proceedings of this conference.

[5] C. Bernard et al.,Phys. Rev. D 77, 014503 (2008) [arXiv:0710.1330 [hep-lat]];

S. Gottlieb, Proceedings of this conference.

[6] Y. Maezawa et al., PoS LAT2007, 207 (2007) [arXiv:0710.0945 [hep-lat]].

[7] S. Ejiri, F. Karsch and K. Redlich, Phys. Lett. B 633, 275 (2006) [arXiv:hep-ph/0509051].

[8] Y. Hatta and T. Ikeda, Phys. Rev. D 67 (2003), 014028, [ArXiv:hep-ph/0210284].

[9] M. Creutz, PoS LAT2006, 208 (2006), [ArXiv:hep-lat/0608020].

[10] M. Creutz, PoS LAT2007, 007 (2007) [arXiv:0708.1295 [hep-lat]].

[11] A. S. Kronfeld, PoS LAT2007, 016 (2007) [arXiv:0711.0699 [hep-lat]].

[12] Y. Shamir, Nucl. Phys. B, 406, 90 (1993), [ArXiv:hep-lat/9303005].

[13] G. Fleming, Ph.D. thesis, [ArXiv:hep-lat/0404020].

[14] P. M. Vranas, Phys. Rev. D 57, 1415 (1998), [ArXiv:hep-lat/9705023].

[15] M. Cheng, Proceedings of this conference; D. Renfrew and P. Vranas, PoS LAT2007, 235 (2007).

[16] G. Aarts and J. Foley [UKQCD Collaboration], JHEP 0702, 062 (2007).

[17] P. Hegde, F. Karsch, E. Laermann and S. Scheredin, Eur. Phys. J., C55, 423 (2008), [ArXiv:0801.4883[hep-lat]].

[18] D. Banerjee, R. V. Gavai and S. Sharma, [ArXiv:0803.3925[hep-lat]]. 